\title{
A microfluidic AFM cantilever based dispensing and aspiration platform
}

\author{
Ralph van Oorschot ${ }^{1 *}$, Hector Hugo Perez Garza ${ }^{2}$, Roy J S Derks ${ }^{1}$, Urs Staufer ${ }^{2}$ and Murali Krishna Ghatkesar ${ }^{2^{*}}$
}

\author{
* Correspondence: \\ r.vanoorschot@ma3solutions.com; \\ m.k.ghatkesar@tudelft.nl \\ ${ }^{1}$ MA3 Solutions, Eindhoven, The \\ Netherlands \\ ${ }^{2}$ Department of Precision and \\ Microsystems Engineering, Delft \\ University of Technology, Delft, The \\ Netherlands
}

\begin{abstract}
We present the development of a microfluidic AFM (atomic force microscope) cantilever-based platform to enable the local dispensing and aspiration of liquid with volumes in the pico-to-femtoliter range. The platform consists of a basic AFM measurement system, microfluidic AFM chip, fluidic interface, automated substrate alignment, external pressure control system and controlled climate near the dispensing area. The microfluidic AFM chip has a hollow silicon dioxide $\left(\mathrm{SiO}_{2}\right)$ cantilever connected to an on-chip fluid reservoir at one end and a slicon nitride $\left(\mathrm{Si}_{3} \mathrm{~N}_{4}\right)$ tip with an aperture on the other end. A 3D printed plastic fluidic interface glued over the on-chip reservoir was used to connect microfluidics and macrofluidics. The fluidics is connected to an external pressure control system ranging from -0.8 bar to 5 bar with 0.1 bar resolution. This pressure range allows dispensing and aspiration of liquids through the cantilever tip aperture. The controlled climate with a temperature control range between $25^{\circ} \mathrm{C}-40^{\circ} \mathrm{C}$ and humidity up to $95 \%$ near the dispensing area keeps the droplets for sufficiently long time before they evaporate. An array of droplets can be programmed to be dispensed automatically and access them again with a position accuracy of 1 micron. Experiments were performed with two types of cantilevers with different geometrical configurations. A minimum flow rate control of $50 \mathrm{fL} / \mathrm{s}$ was obtained and also frequency shift was monitored as the cantilever was filled with liquid. This platform will be used for various chemical and biological applications.
\end{abstract}

Keywords: Microfluidic; Femtoliter; Hollow cantilever; Dispensing; Aspiration; Pressure control system; AFM

\section{Introduction}

The ability to controllably infuse or withdraw different types of reagents with nanometer precision unlocks the door for new processes that can lead to novel opportunities like cell surgery. Lately, more applications, particularly in chemistry and biology are continuously demanding the development of certain tools that would allow the precise dispensing and aspiration of extremely small amounts of liquids at predefined positions. In on-going efforts to achieve this, different types of fluid dispensing devices, such as micropipettes and ink-jet printers, have been explored. Nevertheless, these devices do not offer the degree of precision and control that is required to explore the intended applications. Furthermore, they are limited to handle volumes in the order of micro-to-picoliter [1,2], which constitutes at least three orders of magnitude bigger than the suggested (sub)femtoliter regime for most of the upcoming applications. In attempts to manipulate liquid in the smallest possible regime, scientists have started to 
develop cantilever-based techniques (i.e. DPN [3], NADIS [4], NanoFountain Probe [5] and FluidFM [6]) which have been proved to surpass the picoliter barrier and handle volumes as small as few zeptoliters [7]. These devices, compatible with Atomic Force Microscopy (AFM), combine the versatility of microfluidics with the high precision provided by the cantilever [6]. The challenge, nevertheless, remains on achieving controlled dispensing of the desired volume despite the complications given by external factors such as evaporation [8], humidity [9], hydrophobicity of the tip [10], surface energy of the substrate [10] and viscosity of the liquid [9]. Additionally, the incapability to aspirate controlled amounts of liquid has slowed down the potential break-through applications. In order to overcome these challenges and get closer towards the envisioned goals, we have developed a microcantilever AFM-based platform to enable the dispensing and aspiration of liquids with volumes in the femtoliter range under controlled environment. The various parts in this system constitute a basic AFM measurement system, a microfluidic chip, an automated substrate alignment, a humidity chamber and a microfluidic interface connected to an external pressure control system. The microfluidic chip consisted of a hollow silicon nitride $\left(\mathrm{Si}_{3} \mathrm{~N}_{4}\right)$ tip and channelled silicon dioxide $\left(\mathrm{SiO}_{2}\right)$ cantilever which were connected to a fluidic reservoir located in the handling part of the chip. After filling the reservoir with liquid, a positive or negative pressure relative to the ambient is applied in the reservoir to infuse or withdraw liquids through an aperture located at the apex of the tip. Constant volume systems such as a syringe pump used for applying pressure differences suffer from the effect of shock waves produced by the stepper motors and have a slow response at low flow rates. Moreover, it can take up to few minutes for the pressure to stabilize. Therefore, we have chosen the constant pressure system which is pulse free, has a fast response and can also produce high flow rates. The results shown here represent an improvement over our earlier work on thermal pumping [8], evaporation based pumping and aspiration [11] and syringe-pump based pipetting [12].

\section{Results and discussion Microfluidic chip}

The fabrication of the microfluidic chips resulted in a chip of $1.5 \mathrm{~mm} \times 3 \mathrm{~mm}$, connecting a fluidic reservoir located on the front side of the chip, with storage capacity of $19.5 \mathrm{~nL}$, to the hollow cantilever located on the backside. The cantilever had a total length of $155 \mu \mathrm{m}$. Two cantilever types used are shown in Figure 1. Type-A had an outer width of $42 \mu \mathrm{m}$ (Figure 1a), while type-B had two legs in parallel with an outer width of $6.4 \mu \mathrm{m}$ (Figure 1b). The hollow channels had a width of $3.7 \mu \mathrm{m}$ and a height of $2.2 \mu \mathrm{m}$. The $\mathrm{SiO}_{2}$ walls were measured to be $1.5 \mu \mathrm{m}$. Similarly, the hollow $\mathrm{Si}_{3} \mathrm{~N}_{4}$ tip had a thickness of $200 \mathrm{~nm}$ and a height of $5.65 \mu \mathrm{m}$, over which an aperture of $750 \mathrm{~nm}$ was milled. Overall, these dimensions resulted in a spring constant of $2.4 \mathrm{~N} / \mathrm{m}$ for type-A and $9.4 \mathrm{~N} / \mathrm{m}$ for type-B.

\section{Fluid filling and substrate stage}

We found that when mounting and gluing the microfluidic chip to the plastic interface, which are both mountable in standard AFMs, the maximum pressure that can withstand before detaching was $>5$ bar. The system allowed to regulate the pressure 


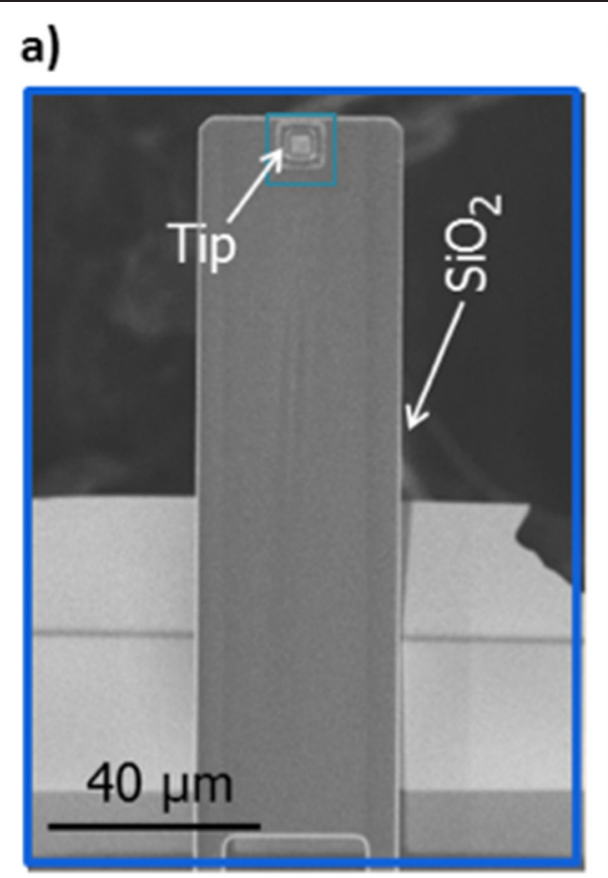

\section{b)}
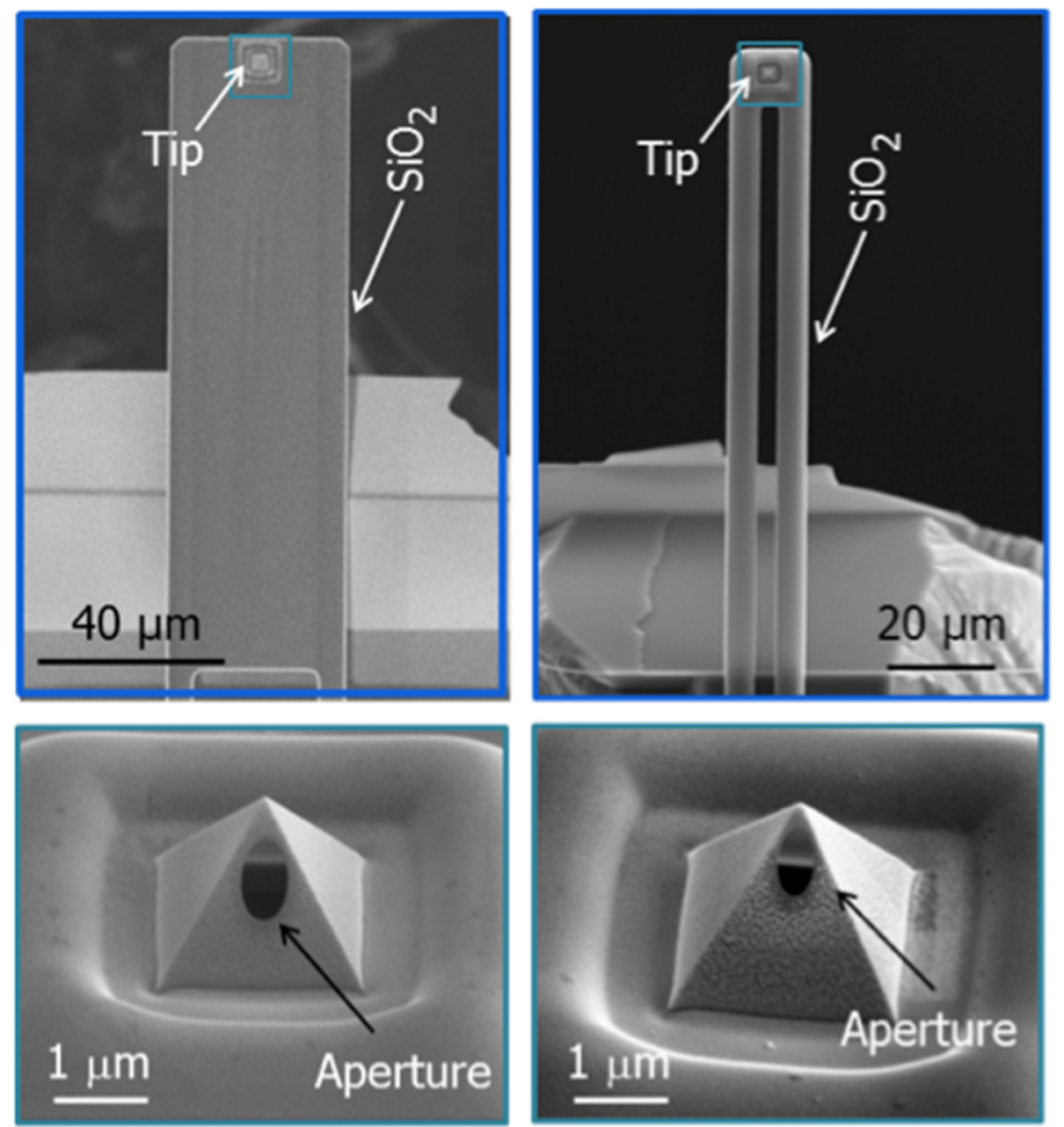

Figure 1 Cantilever types. The chip contains an on-chip fluidic reservoir on the top side, which leads to the fluidic system and the hollow cantilever located on the back side of the chip. Although the microfabrication process was the same, two different types of hollow cantilevers were used for these experiments: (a) type-A had an outer width of $42 \mu \mathrm{m}$ and (b) type-B had two legs with an outer width of $6.4 \mu \mathrm{m}$ each. In both cases, the hollow $\mathrm{Si}_{3} \mathrm{~N}_{4}$ tip had a wall thickness of $200 \mathrm{~nm}$, over which an aperture of $750 \mathrm{~nm}$ was milled.

between -0.8 bars and 5.0 bars with better than 0.1 bar resolution, while a valve accurately timed can switch between the regulator set point and ambient pressure within $100 \mathrm{~ms}$.

The temperature and humidity control resulted in precise regulation of temperature between $25-40^{\circ} \mathrm{C}$ and humidity up to saturation point. Some condensation was noticed around saturation point at high humidity and temperature.

As a first experiment, a chip containing an array of type-B hollow cantilevers independently connected to the fluid reservoir was chosen. A $750 \mathrm{~nm}$ aperture was made on the center cantilever. A $3 \mathrm{~cm}^{3}$ syringe, used as an external liquid reservoir that was filled with deionized water and connected to the pressure system. As a pressure of 1.5bars was applied for 2 minutes, the cantilever was filled and the liquid started to flow out of the aperture as shown in the Figure 2a. The flow speed of the liquid 

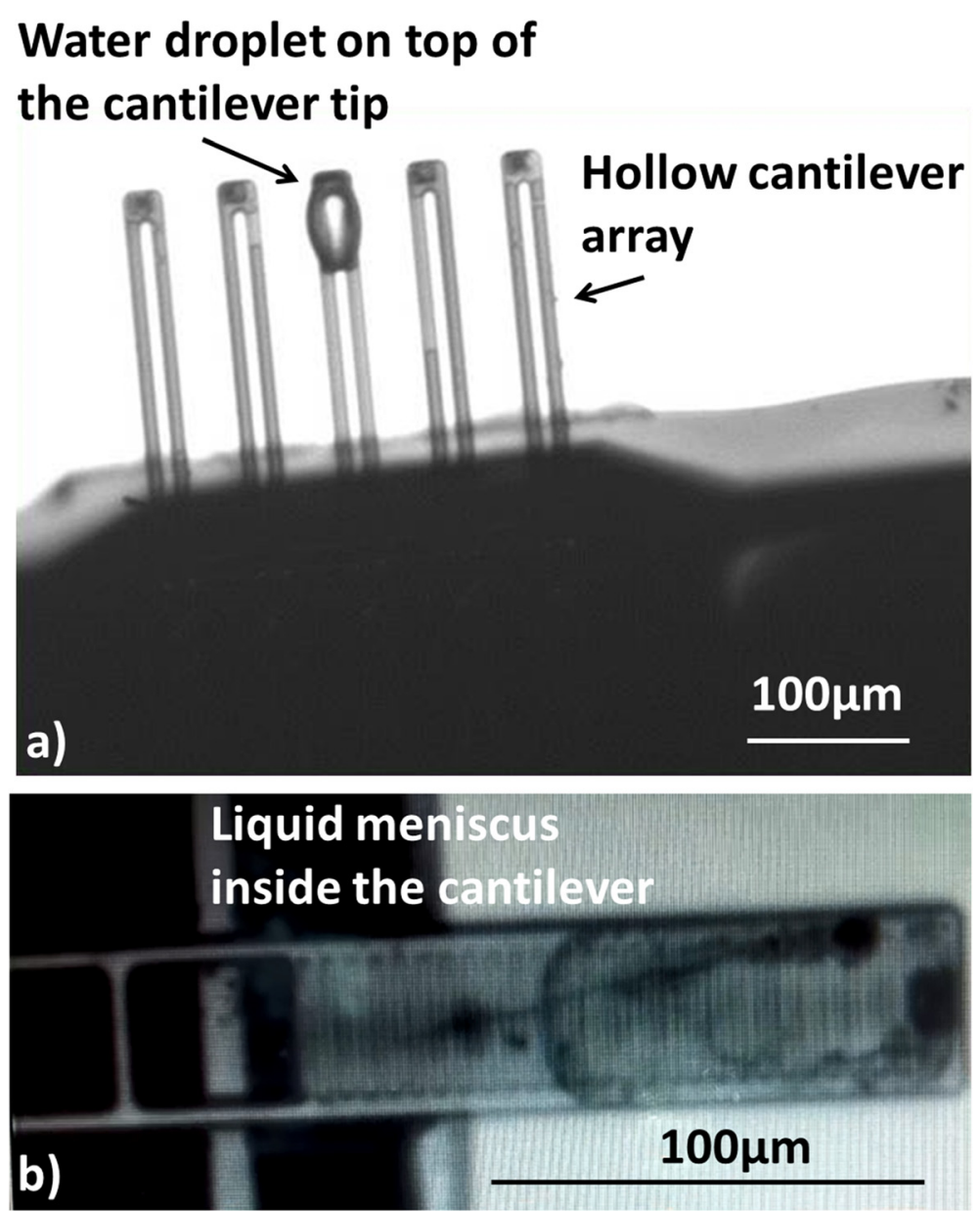

Figure 2 Fluid manipulation inside the cantilever. (a) A chip containing 5 independent type-B cantilevers was used for the initial pipetting experiments. Aperture was made only on the middle cantilever. Applying positive pressure, water was filled in the middle hollow AFM cantilever, and subsequently a droplet was formed outside the tip aperture. The droplet on the cantilever was subsequently aspirated back. (b) Type-A chip is bigger, hence higher volume are uptaken. Liquid meniscus can be seen inside the hollow cantilever as it was getting filled.

was determined by following an air bubble inside the fluidic channels and the minimum rate was found to be $50 \mathrm{fL} / \mathrm{s}$. The liquid was eventually aspirated back into the cantilever by switching to vacuum from pressure.

For type-A cantilevers the resonance frequency shift as the fluid was filled (Figure 2b) inside the hollow cantilever was noted. For each pressure increase step, the liquid meniscus advancement was noted, At 1.2 bar of applied external pressure, the entire cantilever was filled. At appropriate applied pressure, equilibrium was established between evaporation of the droplet at the tip and the supplied liquid.

Silicon oxide chips with a $100 \mu \mathrm{m}$ grid were positioned on the platform and their edges were measured and used as reference by the AFM. We found a re-position accuracy with a standard deviation $<2 \mu \mathrm{m}$ when the substrate was removed and placed back. Similarly, we obtained an accuracy of $<1 \mu \mathrm{m}$ when moving between positions on a substrate. All the characteristics of the platform are given in Table 1. 
Table 1 Characteristics of the hollow cantilever and microfluidic cantilever-based platform

\begin{tabular}{|c|c|}
\hline Parameter & Units \\
\hline \multicolumn{2}{|l|}{ Cantilever } \\
\hline Cantilever dimensions (each leg) & 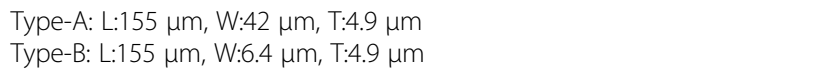 \\
\hline Channel dimensions (each leg) & $\begin{array}{l}\text { Type-A: L:153.5 } \mu \mathrm{m}, \mathrm{W}: 40 \mu \mathrm{m}, \mathrm{T}: 2.2 \mu \mathrm{m} \\
\text { Type-B: L:153.5 } \mu \mathrm{m}, \mathrm{W}: 3.7 \mu \mathrm{m}, \mathrm{T}: 2.2 \mu \mathrm{m}\end{array}$ \\
\hline Spring constant & Type-A: $2.4 \mathrm{~N} / \mathrm{m}$ (when empty); Type-B: $9.4 \mathrm{~N} / \mathrm{m}$ (when empty) \\
\hline Resonance frequency & Type-A: 153.94 kHz (when empty); Type-B: 110 kHz (when empty) \\
\hline Aspiration and dispensing flow rate & 50 fL/s@1.5 bars \\
\hline \multicolumn{2}{|l|}{ External dispenser } \\
\hline Pressure range & 0.85 bars under pressure to 5.0 bar overpressure; 0.01 bar resolution \\
\hline Settling time at dispenser out & $<100 \mathrm{~ms}$ \\
\hline \multicolumn{2}{|l|}{ Climate control } \\
\hline Temperature range & Ambient to $40^{\circ} \mathrm{C} \pm 0.5$ \\
\hline Humidity range & $30 \%-90 \% \mathrm{RH} \pm 5 \%$ non condensing $90 \%-100 \% \mathrm{RH} \pm 5 \%$ condensation \\
\hline \multicolumn{2}{|l|}{ Substrate alignment } \\
\hline Alignment accuracy & $\begin{array}{l}\text { Between chip positions on the same substrate: }<1 \mu \mathrm{m} \text { Between different } \\
\text { chips: }<2 \mu \mathrm{m}\end{array}$ \\
\hline
\end{tabular}

\section{Conclusion}

To conclude, we have developed a hollow AFM cantilever-based platform able to dispense and aspirate liquids in the picoliter to femtoliter volumes of liquid. Besides regular AFM measurements, the AFM chips and the system are designed to handle these liquid volumes in a controlled climate suitable for numerous chemical and biological applications.

\section{Methods and materials}

\section{Microfluidic chip and fluidic interface}

The microfabrication of the microfluidic chip and the characterization of the device have been reported elsewhere [12,13]. Briefly, one wafer was $\mathrm{KOH}$-etched to create the fluidic reservoir (Figures 3a-1), while another was DRIE-etched (Figures 3a-2) in order to pattern the cantilever. Then both wafers were bonded together (Figure 3a) and treated in a wet oxidation furnace. Consequently, the $\mathrm{O}_{2}$ gas entered through the fluidic reservoir and started growing oxide in the patterned cantilever (Figures 3a-4). This resulted in a hollow $\mathrm{SiO}_{2}$ structure that was buried inside the silicon, which was further removed by wet-etching it in $\mathrm{KOH}$ (Figures 3a-5). Later, Focused Ion Beam (FIB) was used to mill the aperture on the tip. For experiments, two different cantilever-designs were used in this paper denoted as rectangular type A and U-shaped type B. Each cantilever differed in the final dimensions, but the fabrication steps were the same.

In order to connect the hollow cantilever to the pressure control system and avoid leakage during liquid transport, a microfluidic plastic interface was manufactured. SolidWorks Software was used to create a suitable model (Figure 3b), which was later printed out of HTM140 polymer using Objet 3D-printer. The polymer has good temperature resistance and high tensile strength. 


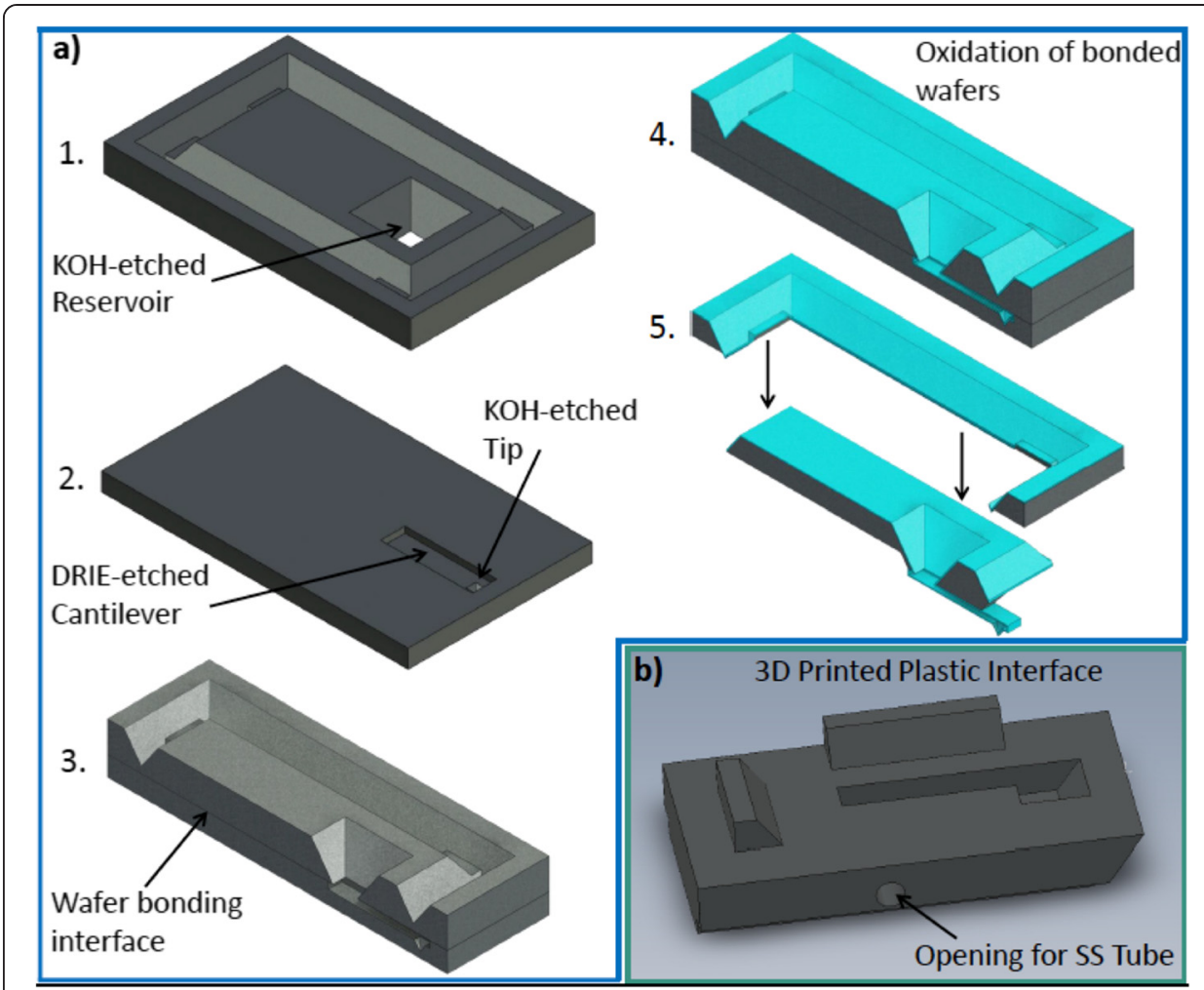

Figure 3 Fabrication of the microfluidic chip and fluidic interface. (a) Schematic of the micro fabrication steps required to create the hollow cantilever. (b) Schematic of the 3D printed fluidic interface with projected alignment guides.

\section{Bonding the microfluidic chip to the interface}

The fluidic interface is designed with alignment guides to self align on the AFM cantilever chip. Before gluing the interface to the chip, a stainless steel (SS) tube was inserted from the side into the interface and glued with Norland Optical Adhesive $86 \mathrm{H}$. The tube had a length of $12.5 \mathrm{~mm}$, an outer diameter of $240 \mu \mathrm{m}$ and inner diameter of $100 \mu \mathrm{m}$. The tube was further connected to external pressure control system using Tygon tubing. The cantilever chip was then glued to the resulting plastic interface using the same glue. The glue could be cured either by UV light or temperature or both. The chip could be easily debonded from the microfluidic interface by dipping in acetone for few seconds without leaving residues. Thus, making the chip and interface reusable. To avoid excessive amount of glue and prevent clogging of the fluidic reservoir during the bonding, the glue was dispensed in controlled amounts using a foot pedal operated pressure-time based dispensing system, FISNAR Inc., Model SL101N at a dispense time of $50 \mathrm{~ms}$ at 0.5 bar. The same glue was dispensed using 30 gauge EFD Nordson dispensing tip with an outer diameter of $310 \mu \mathrm{m}$ (see Figure 4a). After mounting and aligning, the glue was pre-cured by UV light for 10 seconds, using EFOS ACTICURE A4000 with a $100 \mathrm{~W}$ mercury vapor short arc light bulb which produces light in the spectral range of $250-450 \mathrm{~nm}$. To prevent uncured glue which could potentially block the fluidic channels, the parts are cured in a convection oven at $125^{\circ} \mathrm{C}$ for 15 minutes (Figure $4 \mathrm{~b}$ ). 

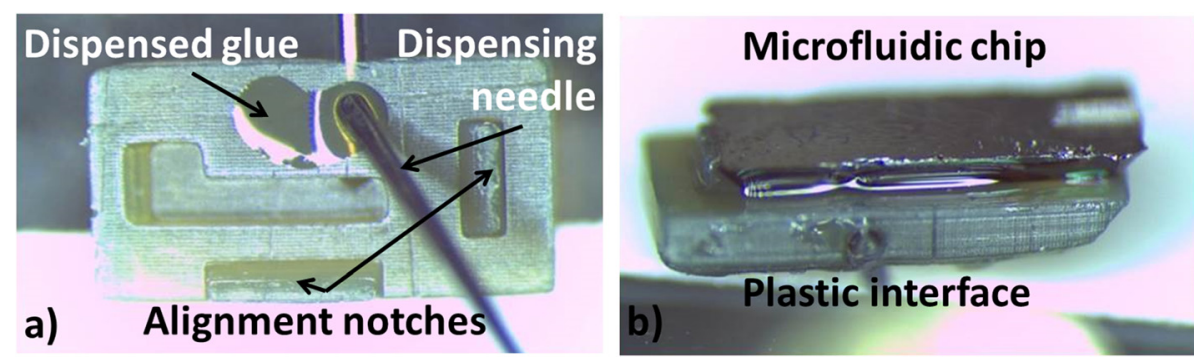

Figure 4 Bonding the cantilever chip to the plastic interface. (a) Top view of the fluidic interface. To avoid excessive amount of glue and prevent the fluidic reservoir of the hollow cantilever from clogging, the correct amount of glue was dispensed. (b) Side view of the fluidic interface and chip after gluing. The chip was aligned against the two alignment notches found on the plastic interface.

\section{Microfluidic AFM dispensing and aspiration platform}

The system is based on the TT-AFM from AFM workshop company with external peripherals like pressure control, climate control and automated $x-y$ stage for substrate alignment attached to the system. A schematic representation and the corresponding photograph of the entire platform is shown in Figure 5.

\section{External pressure control system}

The schematic of the pressure control system is shown in Figure 6. The pressure regulators and the valves control were USB-interfaced via National Instrument NI-6008 interface to the computer. The system includes two MAC valves, type 35A-ACA-DDAA-1BA, which have a switch time of 2-6 ms. Valve-1 switches between pressure and vacuum, while valve- 2 switches between regulated and ambient pressure. Both valves have a fast response. A 3 cc syringe is used as a reservoir, which is located at the left side of the platform, see Figure $5 \mathrm{~b}$. The pressure and duration of a dispense/aspirate action can be defined in the software application.

\section{Temperature and humidity control}

Typically, a droplet in the range of $10 \mu \mathrm{m}^{3}$ (10 fL) or smaller takes less than $1 \mathrm{~s}$ to evaporate [14]. To minimize the evaporation of the droplets and increase the droplet evaporation
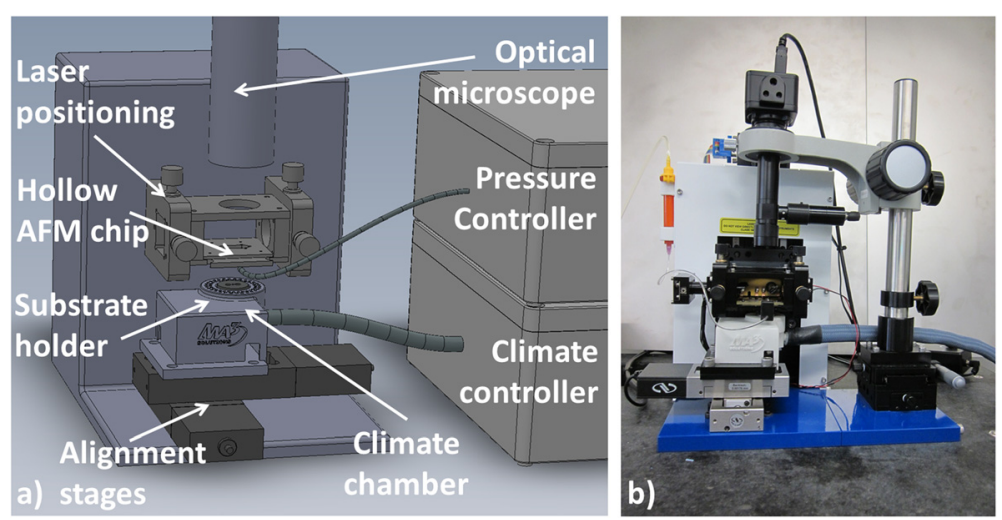

Figure 5 Microfluidic AFM cantilever based dispensing and aspiration platform. (a) Schematic representation (b) Photo of the actual set-up. 


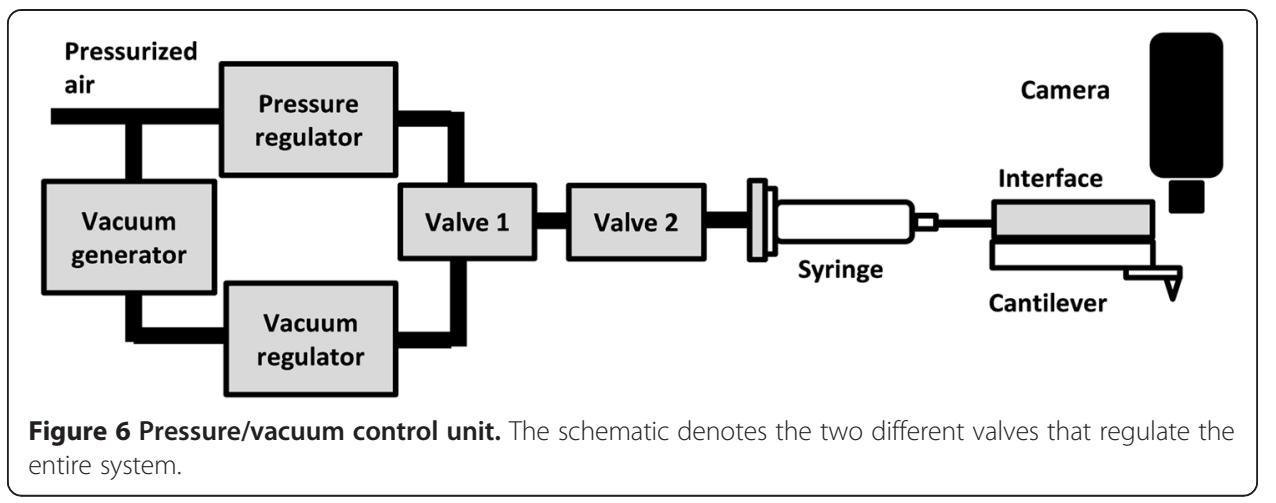

time a controllable climate is required. A semi-open climate chamber is designed for convenient access of the sample substrate, while maintaining the climate around the droplet on the sample. A ring of nozzles ensure to produce a curtain of air with the desired temperature and humidity. The airflow is kept sufficiently high such that diffusion of moisture is slower than the supply of the prepared air. This model is validated with a Finite Element Method (FEM) analysis shown in Figure 7.

To supply air with a controlled temperature and humidity, compressed air is pushed through a bubbler (water bath) and a heated tube. The function of the bubbler is to humidify the air to its saturation point. The air temperature is controlled by heating or cooling the water, which is done by two peltiers-elements that can add or extract energy. This saturated air, at a controlled temperature, flows through a heated tube to increase the temperature and therefore decrease the relative humidity (see Figure 8 ). The

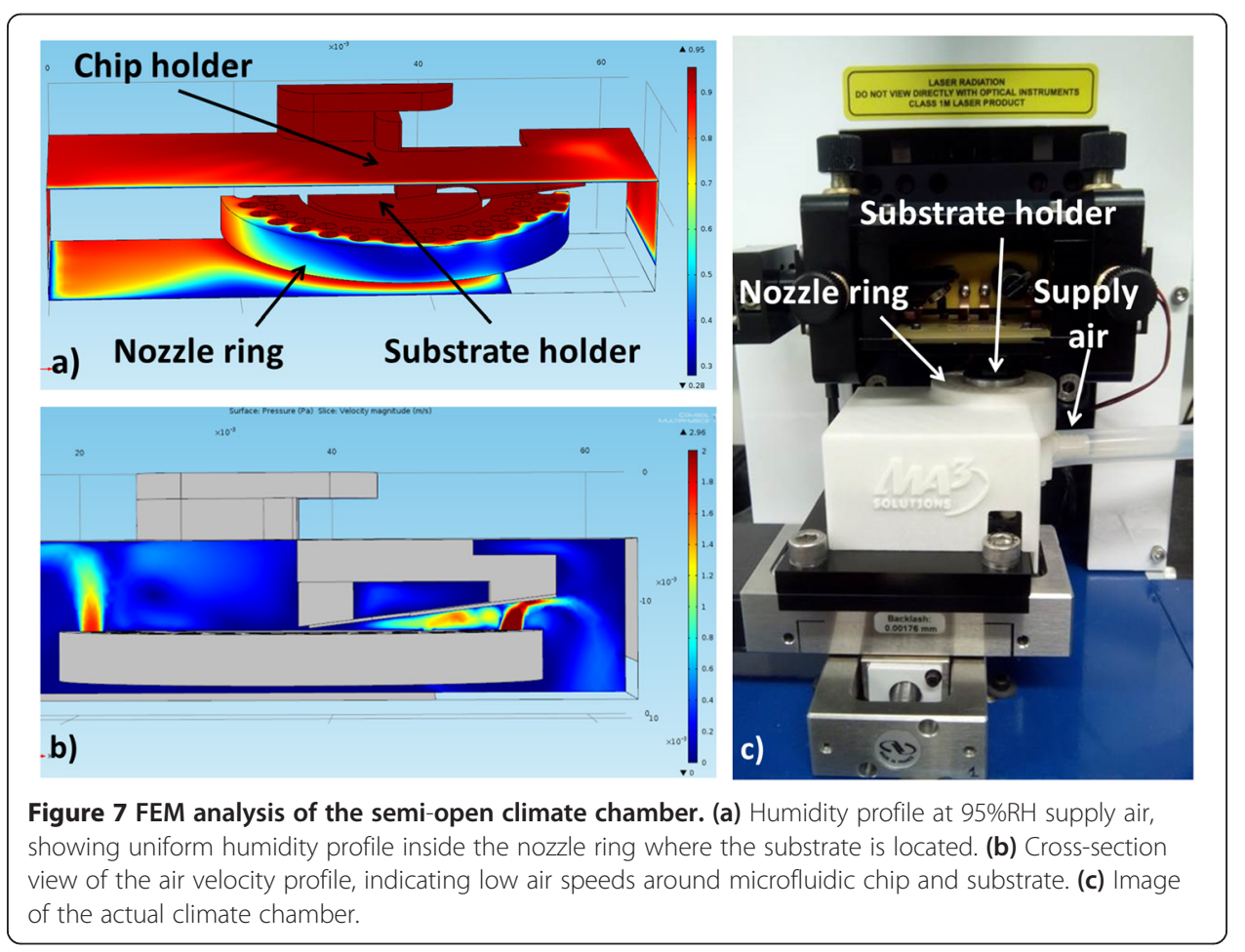




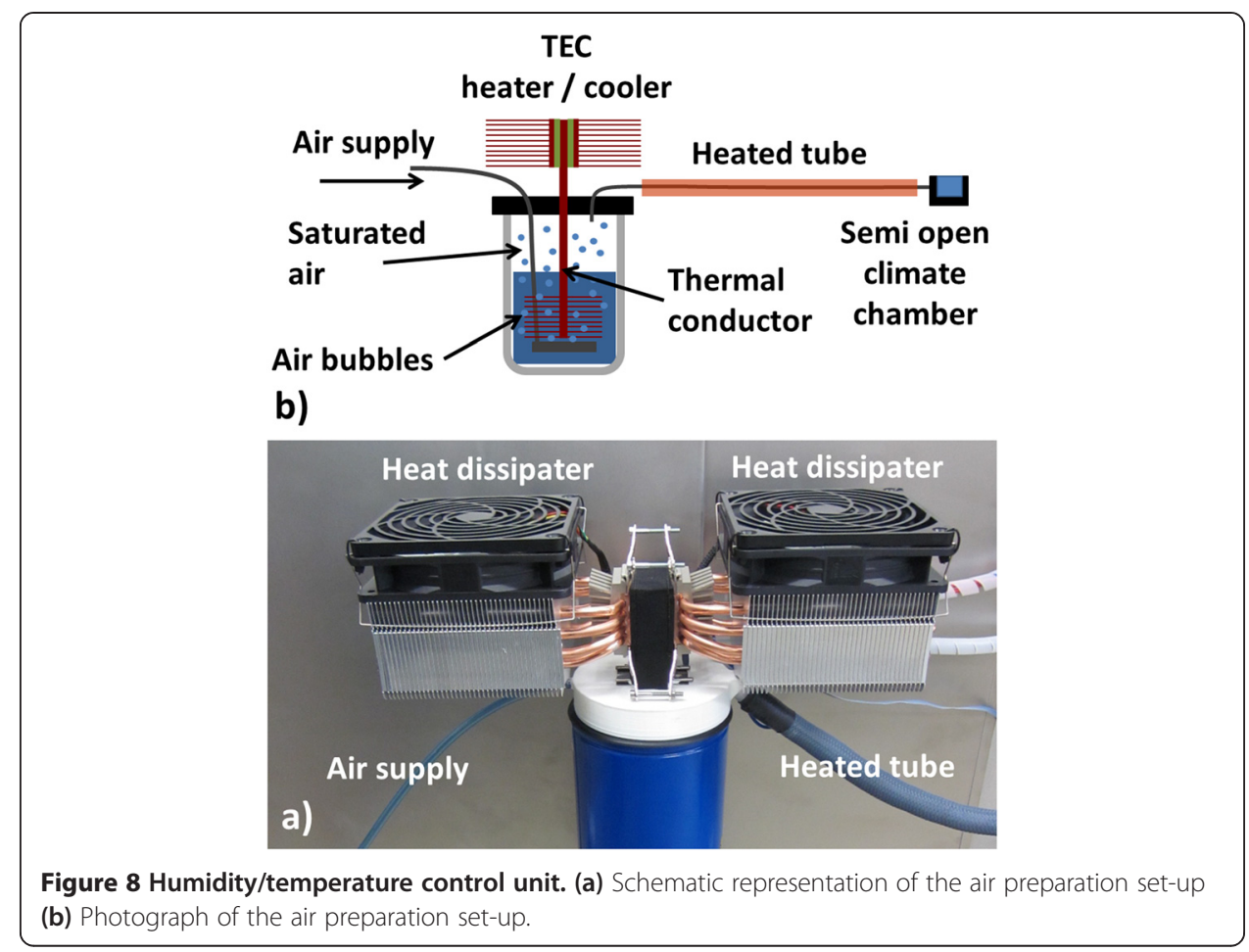

software has two PID control loops: 1) Climate chamber temperature is used as an input for the control setpoint of the tube heating, 2) Climate chamber humidity is used as an input for the water temperature setpoint. Note that this setup only increases the droplet lifetime significantly based on the humidity value chosen (upto 95\% noncondensing) but doesn't prevent completely.

\section{Substrate to tip alignment}

Two Newport SMC100 stages with a range of $25 \mathrm{~mm}$ were mounted under the substrate (Figure 5a). These stages are used for XY positioning the substrate and the chip. Silicon oxide chips from Bioforce with a $100 \mu \mathrm{m}$ square grid were used to align on. The edges of these gridlines were detected with machine vision and software determined the tip and substrate position. The software could position the tip in one of the squares and select a position within this square. This could be used to find back a previously dispensed droplet within an accuracy of 1 micron. After positioning, the AFM piezo stages could be used for sub-micron positioning.

\section{Dispense automation software}

A Labview based software program was developed which is able to handle automatic dispensing tasks. Each dispensing action consisted of the following steps: the tip deflection check and a small Z-step. When the deflection was measured, the software stored the corresponding substrate height and moved a defined number of steps towards the substrate. Then the dispense action was performed and the tip was moved back away from the substrate. Figure 9 shows the software controllable parameters. In automatic mode, the software positions the tip to a new XY position and the process is repeated. An array of points can be given to the software via a CSV file. 

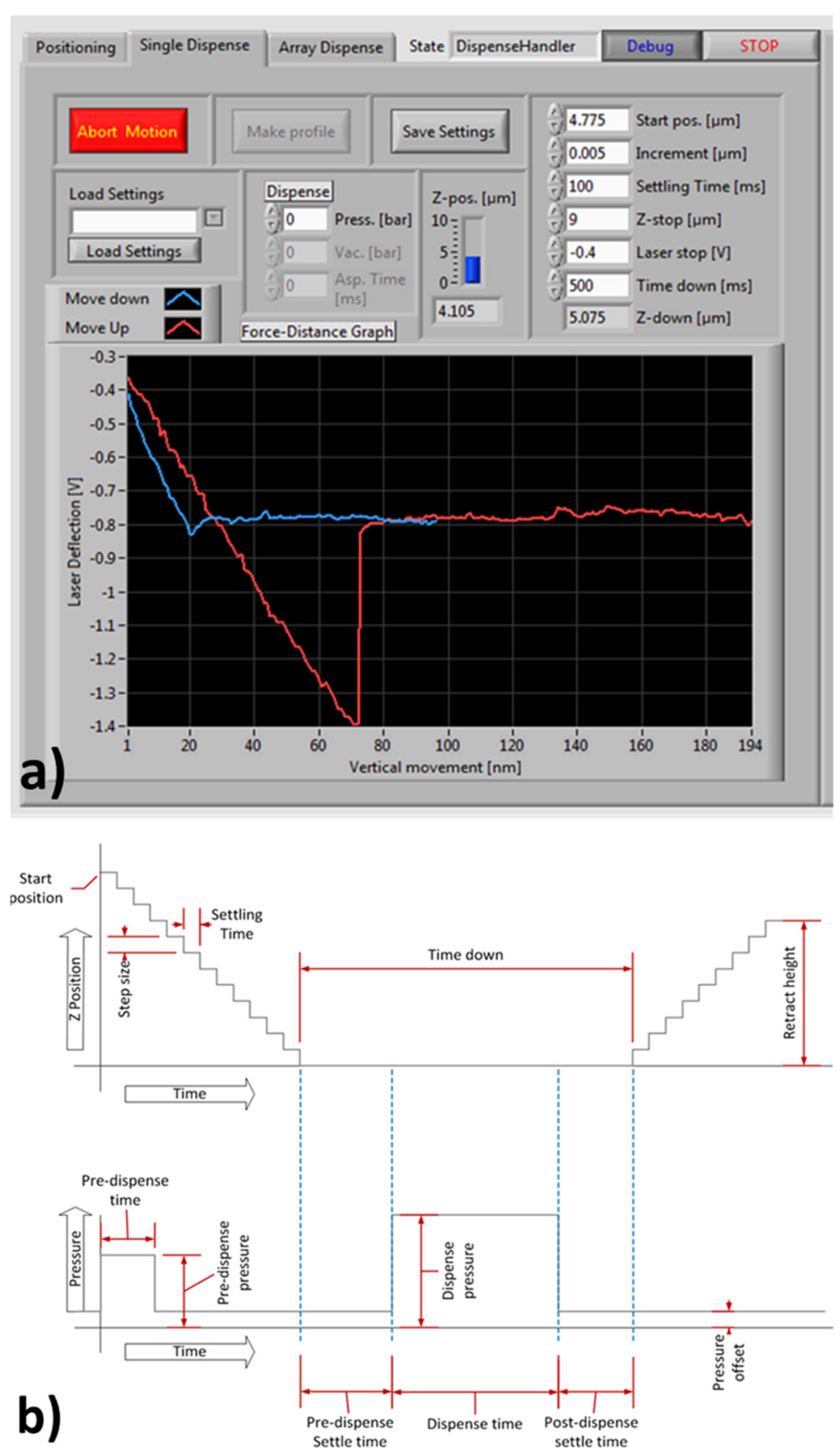

Figure 9 Automation software screenshots. a) Dispensing software, showing a force distance curve a droplet is a height of approximate $50 \mathrm{~nm}(\sim 3.5 \mathrm{fL})$, b) Controllable parameters, with the Z-position settings (top panel) and dispense pressure settings (bottom panel).

\section{Competing interest}

The authors declare that they have no competing interest.

\section{Authors' contribution}

RVO and RJSD carried out the implementation of the microfluidic interface and the pheripherals involved in the dispensing and aspiration platform development. HHPG fabricated the microfluidic AFM device. US and MKG were involved in the design of the project. RvO, HHPG, MKG were involved in the experiments and interpretation of the results. All the authors contributed to the writing of the manuscript. All authors read and approved the final manuscript. 


\section{Acknowledgements}

This work is supported by NanoNextNL, a micro and nanotechnology consortium of the government of the Netherlands and 130 partners

Received: 17 June 2014 Accepted: 24 December 2014

Published online: 12 March 2015

\section{References}

1. Elkins KM. Academic Press Publications; 2013.

2. Hutchings IMaM. Hoboken, New Jersey: G.D., John Wiley \& Sons; 2013

3. Piner RD, Zhu J, Xu F, Hong SH, Mirkin CA. Science. 1999; 283:661-3.

4. Meister A, Jeney S, Liley M, Akiyama T, Staufer U, de Rooij NF, et al. Microelectron Eng. 2003;67-8:644-50

5. Kim KH, Moldovan N, Ke C, Espinosa HD. Micro- and Nanosystems. 2004; 782:267-72.

6. Meister A, Gabi M, Behr P, Studer P, Voros J, Niedermann P, et al. Nano Lett. 2009; 9:2501-7.

7. Kaisei K, Satoh N, Kobayashi K, Matsushige K, Yamada H. Nanotechnology. 2011; 22:175301.

8. Perez Garza HH, Ghatkesar M, Staufer U. Journal of Micro-Bio. Robotics. 2013;8:33-40.

9. Fang AP, Dujardin E, Ondarcuhu T. Nano Lett. 2006; 6:2368-74.

10. Fang AP, Dujardin E, Ondarcuhu T. J Phys Conf Ser. 2007;61:298-301.

11. Perez Garza HH, Ghatkesar M, Staufer U. Micro Nano Lett. 2013; 8:758-61.

12. Ghatkesar MK, Perez Garza HH, Staufer U. Microelectron Eng. 2014; 124:22-5.

13. HugTS, Biss T, de Rooij NF, Staufer Q. Transducers '05, Digest of Technical Papers. 2005; 1 and 2:1191-4.

14. Bonaccurso E, Golovko DS, Bonanno P, Raiteri R, Haschke T, Wiechert W, et al. Atomic Force Microscope Cantilevers Used as Sensors for Monitoring Microdrop Evaporation, Applied Scanning Probe Methods XI. Berlin Heidelberg, Germany: Springer; 2009. p. 17-38.

Submit your manuscript to a SpringerOpen ${ }^{\circ}$ journal and benefit from:

- Convenient online submission

- Rigorous peer review

- Immediate publication on acceptance

- Open access: articles freely available online

- High visibility within the field

- Retaining the copyright to your article 